\title{
Adaptive Iterative Learning Control for Complex Dynamical Networks
}

\author{
Xiuqing $\mathrm{Hao}^{1,2}$ and Junmin $\mathrm{Li}^{1}$ \\ ${ }^{1}$ School of Mathematics and Statistics, Xidian University, Xi'an 710071, China \\ ${ }^{2}$ School of Science, Xian Shiyou University, Xian 710065, China \\ Correspondence should be addressed to Junmin Li; jmli@mail.xidian.edu.cn
}

Received 17 June 2014; Accepted 17 August 2014

Academic Editor: Dan Wang

Copyright (c) 2015 X. Hao and J. Li. This is an open access article distributed under the Creative Commons Attribution License, which permits unrestricted use, distribution, and reproduction in any medium, provided the original work is properly cited.

\begin{abstract}
A new adaptive iterative learning control scheme is proposed for complex dynamical networks with repetitive operation over a fixed time interval. By designing difference type updating laws for unknown time-varying parameters and coupling strength, the state of each node in complex dynamical networks can track the reference signal. By constructing a composite energy function, a sufficient condition of the convergence of tracking error sequence is achieved in the iteration domain. Finally, a numerical example is given to show the effectiveness of the designed method.
\end{abstract}

\section{Introduction}

Iterative learning control (ILC) is an effective control scheme suitable for dynamical systems with repetitive operation over a fixed time interval. It has received considerable attentions since it was introduced by Arimoto et al. [1]. The ILC system improves its control performance by updating the control input iteratively with the information of errors and inputs in the preceding trials. In view of methodology, the ILC can be classified into the PID-type algorithm and the adaptive iterative learning control (AILC) approach.

Up to now, AILC schemes have been widely used in nonlinear systems [2-10]. Using a composite energy function, $\mathrm{Xu}$ and Tan [3] proposed an adaptive learning control approach for nonlinear systems with time-varying parametric uncertainties. Chen and Zhang [5] designed an AILC scheme for nonlinearly parameterized systems with unknown time-varying delays. An adaptive ILC for a class of discrete-time systems with iteration-varying trajectory and random initial condition was shown in [8]. The AILC of systems with unknown control direction was proposed in $[9,10]$. In view of control objective, the AILC system can track the iteration-invariant reference signal $[2-6,9,10]$ and the iteration-variant one $[7,8]$.
Complex dynamical networks [11-14] exist in many fields such as science, nature, and human societies. A complex dynamical network is a large set of interconnected nodes, in which each node represents an individual element and the connection between every two nodes represents the relation. In this context, a complex dynamical network is a class of interconnected system, in which each node is a subsystem. Synchronization of complex dynamical networks is an important topic that has drawn a great deal of attention [15-23]. Various control schemes, such as impulsive control [15-17], pinning control [17, 18, 22], and adaptive control [19-22], were reported to achieve network synchronization. Many synchronization criteria have been derived for complex dynamical networks with different special features, such as time-delay [19-21], time-varying coupling parameter [20, 21], nonlinear coupling [22, 23], and nonidentical nodes [23]. It is worth noting that the tracking problem of complex dynamical networks is rarely mentioned.

Just as mentioned above, a complex dynamical network is a large-scale interconnected system. Some control schemes were designed for the stabilization and tracking of largescale interconnected systems, such as decentralized adaptive robust control [24] and decentralized iterative learning control [25]. Thus, it is meaningful to apply the iterative learning 
control strategy to complex dynamical networks with repetitive operation over a fixed time interval.

Motivated by the above discussions, a new adaptive iterative learning control scheme is proposed for complex dynamical networks with nonidentical nodes and unknown time-varying parameters in this paper. The control objective is that the state of each node in complex dynamical networks can track the desired trajectory over a fixed time interval through the iterative learning process. As we all know, the network topology of complex dynamical networks is the difficulty in the control design. Compared with the simple plant without network connection, the controllers of complex dynamical networks are more complicated. In this paper, a simple feedback controller is designed by analyzing the network topology of complex dynamical networks, which makes the control scheme more simple and useful.

The rest of this paper is organized as follows. The problem formulation and preliminaries are given in Section 2. Section 3 gives the design of controllers and adaptive learning laws. In Section 4, the convergence property of the proposed adaptive iterative learning control method is given. In Section 5, an illustrative example is given to show the effectiveness of the designed method. Finally, conclusions are given in Section 6.

\section{Problem Formulation and Preliminaries}

Consider a controlled complex dynamical network consisting of $N$ nonidentical nodes and operating repetitively over a fixed time interval $[0, T]$. The state equation of each node is given by

$$
\begin{aligned}
\dot{\mathbf{x}}_{i}^{(k)}(t)= & \theta_{i}(t) \boldsymbol{\xi}_{i}\left(\mathbf{x}_{i}^{(k)}(t)\right) \\
& +\eta(t) \sum_{j=1}^{N} a_{i j} \Gamma \mathbf{x}_{j}^{(k)}(t)+\mathbf{u}_{i}^{(k)}(t), \quad i=1,2, \ldots, N
\end{aligned}
$$

where the superscript $k$ denotes the iteration number of learning, $\mathbf{x}_{i}^{(k)}(t)=\left(x_{i 1}^{(k)}(t), x_{i 2}^{(k)}(t), \ldots, x_{i n}^{(k)}(t)\right)^{T} \in \mathbf{R}^{n}$ represents the state vector, $\boldsymbol{\xi}_{i}\left(\mathbf{x}_{i}^{(k)}(t)\right) \in \mathbf{R}^{n}$ is a known smooth nonlinear vector-valued function, and $\mathbf{u}_{i}^{(k)}(t)=\left(u_{i 1}^{(k)}(t)\right.$, $\left.u_{i 2}^{(k)}(t), \ldots, u_{i n}^{(k)}(t)\right)^{T} \in \mathbf{R}^{n}$ denotes the input. $\boldsymbol{\Gamma}=\left(\gamma_{i j}\right)_{n \times n} \in$ $R^{n \times n}$ is the constant inner-coupling matrix; $\mathbf{A}=\left(a_{i j}\right)_{N \times N} \in$ $\mathbf{R}^{N \times N}$ is the outer-coupling matrix, in which $a_{i j}$ is defined as follows: if there is a connection between node $i$ and node $j$ $(j \neq i)$, then $a_{i j} \neq 0$, else $a_{i j}=0$, and the diagonal elements of matrix $\mathbf{A}$ are defined by

$$
a_{i i}=-\sum_{j=1, j \neq i}^{N} a_{i j}, \quad i=1,2, \ldots, N .
$$

In network (1), $\theta_{i}(t)$ is an unknown continuous time-varying parameter, which shows that there are some unknown terms in the dynamical equation of each node; $\eta(t)$ is the unknown continuous time-varying coupling strength of the network.
Remark 1 . The unknown parameters are widely considered in the analysis and control of systems. In this paper, the unknown parameters in the complex dynamical network make the network study as real as possible.

The control objective is to find a sequence of control input $\mathbf{u}_{i}^{(k)}(t)$ on $[0, T]$ and the updating laws for unknown timevarying parameters of each node, such that the tracking error sequence $\mathbf{e}_{i}^{(k)}(t)$ converges to zero as $k$ tends to infinity; that is,

$$
\lim _{k \rightarrow \infty} \mathbf{e}_{i}^{(k)}(t)=\lim _{k \rightarrow \infty}\left[\mathbf{x}_{i}^{(k)}(t)-\mathbf{s}(t)\right]=0, \quad i=1,2, \ldots, N,
$$

where the tracking error sequence is $\mathbf{e}_{i}^{(k)}(t)=\mathbf{x}_{i}^{(k)}(t)-\mathbf{s}(t)=$ $\left(e_{i 1}^{(k)}(t), e_{i 2}^{(k)}(t), \ldots, e_{i n}^{(k)}(t)\right)^{T}$ with the given desired trajectory $\mathbf{s}(t)=\left(s_{1}(t), s_{2}(t), \ldots, s_{n}(t)\right)^{T} \in \mathbf{R}^{n}$.

In order to achieve the above objective, the following mathematical preliminaries are needed.

Assumption 2. In network (1), the inner-coupling matrix $\Gamma$ and outer-coupling matrix $\mathbf{A}$ satisfy $\|\boldsymbol{\Gamma}\|=\gamma$ and $\left|a_{i j}\right| \leq a$ for $i, j=1,2, \ldots, N$, where $\gamma$ and $a$ are positive constants.

Assumption 3. The continuous time-varying parameter $\eta(t)$ is nonnegative on $[0, T]$.

Assumption 4. The desired trajectory $\mathbf{s}(t)$ is a differentiable vector-valued function with respect to $t \in[0, T]$, so that the boundedness of it is achieved.

Assumption 5. The initial condition satisfies $\mathbf{e}_{i}^{(k)}(0)=0$; that is, $\mathbf{x}_{i}^{(k)}(0)=\mathbf{s}(0)$ for $i=1,2, \ldots, N$.

Lemma 6 (Young's inequality). For any vectors $\mathbf{x}, \mathbf{y} \in \mathbf{R}^{n}$ and any $\varepsilon>0$, the following matrix inequality holds:

$$
\mathbf{x}^{T} \mathbf{y} \leq \varepsilon \mathbf{x}^{T} \mathbf{x}+\frac{1}{4 \varepsilon} \mathbf{y}^{T} \mathbf{y}
$$

Lemma 7. If the derivative of the sequence $\mathbf{e}_{i}^{(k)}(t)(t \in$ $[0, T])$ is uniformly bounded with respect to $k$ and satisfies $\lim _{k \rightarrow \infty} \int_{0}^{T}\left(\mathbf{e}_{i}^{(k)}(\tau)\right)^{T} \mathbf{e}_{i}^{(k)}(\tau) d \tau=0$, then $\lim _{k \rightarrow \infty} \mathbf{e}_{i}^{(k)}(t)=0$ uniformly on $[0, T]$.

Proof. By invoking the definition of $\mathbf{e}_{i}^{(k)}(t)$ and carefully calculating, we have

$$
\lim _{k \rightarrow \infty} \int_{0}^{T}\left[\mathbf{e}_{i}^{(k)}(\tau)\right]^{T} \mathbf{e}_{i}^{(k)}(\tau) d \tau=\sum_{j=1}^{n} \lim _{k \rightarrow \infty} \int_{0}^{T}\left(e_{i j}^{(k)}(\tau)\right)^{2} d \tau=0,
$$

which yields $\lim _{k \rightarrow \infty} \int_{0}^{T}\left(e_{i j}^{(k)}(\tau)\right)^{2} d \tau=0$ for $j=1,2, \ldots, n$. According to Lemma 2 of [6], we get that $\lim _{k \rightarrow \infty} e_{i j}^{(k)}(t)=0$ uniformly on $[0, T]$. Hence, $\lim _{k \rightarrow \infty} \mathbf{e}_{i}^{(k)}(t)=0$ uniformly on $[0, T]$. 
Definition 8. For a scalar $b$, a saturation function is defined as follows:

$$
\operatorname{sat}(b)= \begin{cases}b_{1}, & b<b_{1} \\ b, & b_{1} \leq b \leq b_{2} \\ b_{2}, & b>b_{2},\end{cases}
$$

where $\left\{b_{1}, b_{2}\right\}$ are the lower and upper bounds of $b$ and satisfy $b_{1}<b_{2}$.

Lemma 9 (see [6]). Given scalars $a$ and $b$, suppose that $b_{1}<$ $a<b_{2}$; then

$$
[a-\operatorname{sat}(b)][b-\operatorname{sat}(b)] \leq 0
$$

\section{Design of Controllers and Adaptive Learning Laws}

In this section, a new adaptive iterative learning control scheme is proposed for the network described by (1). The dynamical equation of tracking error sequence $\mathbf{e}_{i}^{(k)}(t)$ is expressed as follows:

$$
\begin{aligned}
\dot{\mathbf{e}}_{i}^{(k)}(t)= & \theta_{i}(t) \boldsymbol{\xi}_{i}\left(\mathbf{x}_{i}^{(k)}(t)\right) \\
& +\eta(t) \sum_{j=1}^{N} a_{i j} \Gamma \mathbf{e}_{j}^{(k)}(t)+\mathbf{u}_{i}^{(k)}(t)-\dot{\mathbf{s}}(t) .
\end{aligned}
$$
by

To achieve the control objective (3), we design controllers

$$
\begin{aligned}
\mathbf{u}_{i}^{(k)}(t)= & -\widehat{\theta}_{i}^{(k)}(t) \boldsymbol{\xi}_{i}\left(\mathbf{x}_{i}^{(k)}(t)\right) \\
& -\operatorname{Na\gamma }_{\widehat{\eta}^{(k)}}(t) \mathbf{e}_{i}^{(k)}(t)+\dot{\mathbf{s}}(t)-c \mathbf{e}_{i}^{(k)}(t),
\end{aligned}
$$

where $\widehat{\theta}_{i}^{(k)}(t)$ and $\widehat{\eta}^{(k)}(t)$ are estimations to $\theta_{i}(t)$ and $\eta(t)$, respectively.

Remark 10. The designed controller (9) is a simple feedback controller for each node, in which $-\operatorname{Na\gamma \widehat {\eta }^{(k)}}(t) \mathbf{e}_{i}^{(k)}(t)$ is used to deal with the network topology of complex dynamical networks. If we proposed the AILC scheme for systems without network connection, this term is not needed.

Denoting $\widetilde{\theta}_{i}^{(k)}(t)=\theta_{i}(t)-\widehat{\theta}_{i}^{(k)}(t)$ and $\widetilde{\eta}^{(k)}(t)=\eta(t)-\widehat{\eta}^{(k)}(t)$, which are the estimation errors, we obtain the following closed-loop error equation:

$$
\begin{aligned}
\dot{\mathbf{e}}_{i}^{(k)}(t)= & \widetilde{\theta}_{i}^{(k)}(t) \boldsymbol{\xi}_{i}\left(\mathbf{x}_{i}^{(k)}(t)\right) \\
& +\eta(t) \sum_{j=1}^{N} a_{i j} \Gamma \mathbf{e}_{j}^{(k)}(t)-N a \gamma \widehat{\eta}^{(k)}(t) \mathbf{e}_{i}^{(k)}(t)-c \mathbf{e}_{i}^{(k)}(t) .
\end{aligned}
$$

A Lyapunov functional at the $k$ th iteration is selected as

$$
V^{(k)}(t)=\frac{1}{2} \sum_{i=1}^{N}\left(\mathbf{e}_{i}^{(k)}(t)\right)^{T} \mathbf{e}_{i}^{(k)}(t)
$$

The derivative of $V^{(k)}(t)$ is

$$
\begin{aligned}
\dot{V}^{(k)}(t)= & \sum_{i=1}^{N}\left(\mathbf{e}_{i}^{(k)}(t)\right)^{T} \dot{\mathbf{e}}_{i}^{(k)}(t)=-c \sum_{i=1}^{N}\left(\mathbf{e}_{i}^{(k)}(t)\right)^{T} \mathbf{e}_{i}^{(k)}(t) \\
& +\sum_{i=1}^{N}\left(\mathbf{e}_{i}^{(k)}(t)\right)^{T}\left[\widetilde{\theta}_{i}^{(k)}(t) \boldsymbol{\xi}_{i}\left(\mathbf{x}_{i}^{(k)}(t)\right)\right] \\
& -N a \gamma \sum_{i=1}^{N} \widehat{\eta}^{(k)}(t)\left(\mathbf{e}_{i}^{(k)}(t)\right)^{T} \mathbf{e}_{i}^{(k)}(t) \\
& +\sum_{i=1}^{N}\left(\mathbf{e}_{i}^{(k)}(t)\right)^{T} \eta(t) \sum_{j=1}^{N} a_{i j} \mathbf{r e}_{j}^{(k)}(t) .
\end{aligned}
$$

According to Assumptions 2 and 3 and Lemma 6, one obtains

$$
\begin{aligned}
\dot{V}^{(k)}(t) \leq & -c \sum_{i=1}^{N}\left(\mathbf{e}_{i}^{(k)}(t)\right)^{T} \mathbf{e}_{i}^{(k)}(t) \\
& +\sum_{i=1}^{N}\left(\mathbf{e}_{i}^{(k)}(t)\right)^{T}\left[\widetilde{\theta}_{i}^{(k)}(t) \boldsymbol{\xi}_{i}\left(\mathbf{x}_{i}^{(k)}(t)\right)\right] \\
& -N a \gamma \sum_{i=1}^{N} \widehat{\eta}^{(k)}(t)\left(\mathbf{e}_{i}^{(k)}(t)\right)^{T} \mathbf{e}_{i}^{(k)}(t) \\
& +\sum_{i=1}^{N} \eta(t)\left(N a^{2} \varepsilon+\frac{1}{4 \varepsilon} N \gamma^{2}\right)\left(\mathbf{e}_{i}^{(k)}(t)\right)^{T} \mathbf{e}_{i}^{(k)}(t),
\end{aligned}
$$

where $\varepsilon$ is a positive constant. Choosing $\varepsilon=(1 / 2) a^{-1} \gamma$, we have $N a^{2} \varepsilon+(1 / 4 \varepsilon) N \gamma^{2}=N a \gamma$; then

$$
\begin{aligned}
\dot{V}^{(k)}(t) \leq & -c \sum_{i=1}^{N}\left(\mathbf{e}_{i}^{(k)}(t)\right)^{T} \mathbf{e}_{i}^{(k)}(t) \\
& +\sum_{i=1}^{N} \widetilde{\theta}_{i}^{(k)}(t)\left(\mathbf{e}_{i}^{(k)}(t)\right)^{T} \boldsymbol{\xi}_{i}\left(\mathbf{x}_{i}^{(k)}(t)\right) \\
& +N a \gamma \sum_{i=1}^{N} \widetilde{\eta}^{(k)}(t)\left(\mathbf{e}_{i}^{(k)}(t)\right)^{T} \mathbf{e}_{i}^{(k)}(t) .
\end{aligned}
$$
are

The proposed adaptive learning laws of $\widehat{\theta}_{i}^{(k)}(t)$ and $\widehat{\eta}^{(k)}(t)$

$$
\begin{gathered}
\widehat{\theta}_{i}^{(k)}(t)=\operatorname{sat}\left(\widehat{\theta}_{i}^{*(k)}(t)\right), \\
\widehat{\theta}_{i}^{*(k)}(t)=\operatorname{sat}\left(\widehat{\theta}_{i}^{*(k-1)}(t)\right)+q_{i 0}\left(\boldsymbol{\xi}_{i}\left(\mathbf{x}_{i}^{(k)}(t)\right)\right)^{T} \mathbf{e}_{i}^{(k)}(t), \\
\widehat{\theta}_{i}^{*(-1)}(t)=0, \quad i=1,2, \ldots, N, \\
\widehat{\eta}^{(k)}(t)=\operatorname{sat}\left(\widehat{\eta}^{*(k)}(t)\right), \\
\widehat{\eta}^{*(k)}(t)=\operatorname{sat}\left(\widehat{\eta}^{*(k-1)}(t)\right)+q_{1} \sum_{i=1}^{N}\left(\mathbf{e}_{i}^{(k)}(t)\right)^{T} \mathbf{e}_{i}^{(k)}(t), \\
\widehat{\eta}^{*(-1)}(t)=0,
\end{gathered}
$$

where $q_{i 0}$ and $q_{1}$ are positive design parameters. 
The saturation function $\operatorname{sat}(\cdot)$ in (15) is the same as Definition 8; that is, for any signal $y(t)(t \in[0, T])$, we have

$$
\operatorname{sat}(y(t))= \begin{cases}b_{1}, & y(t)<b_{1} \\ y(t), & b_{1} \leq y(t) \leq b_{2} \\ b_{2}, & y(t)>b_{2}\end{cases}
$$

where $\left\{b_{1}, b_{2}\right\}$ are the lower and upper bounds. Without losing generality, we assume that $\theta_{i}(t)$ and $\eta(t)$ lie within the saturation bounds.

\section{Convergence Analysis}

The convergence property of the proposed adaptive iterative learning control scheme is summarized in the following theorem.

Theorem 11. Considering system (1) under Assumptions 2, 3, 4, and 5, the control law (9) and the adaptive learning laws (15) guarantee the convergence of the tracking error sequence $\mathbf{e}_{i}^{(k)}(t)$; that is, $\lim _{k \rightarrow \infty} \mathbf{e}_{i}^{(k)}(t)=0$ for $i=1,2, \ldots, N$. Furthermore, all signals in the closed-loop system are bounded.

Proof. To facilitate the convergence analysis, we choose a Lyapunov-Krasovskii functional at the $k$ th iteration as follows:

$$
\begin{aligned}
W^{(k)}(t)= & V^{(k)}(t)+\sum_{i=1}^{N} \frac{1}{2 q_{i 0}} \int_{0}^{t}\left(\widetilde{\theta}_{i}^{(k)}(\tau)\right)^{2} d \tau \\
& +\frac{N a \gamma}{2 q_{1}} \int_{0}^{t}\left(\widetilde{\eta}^{(k)}(\tau)\right)^{2} d \tau .
\end{aligned}
$$

Firstly, we calculate the difference of $W^{(k)}(t)$ at the $k$ th iteration as follows:

$$
\begin{aligned}
\Delta W^{(k)}(t)= & W^{(k)}(t)-W^{(k-1)}(t)=V^{(k)}(t)-V^{(k-1)}(t) \\
& +\sum_{i=1}^{N} \frac{1}{2 q_{i 0}} \int_{0}^{t}\left[\left(\widetilde{\theta}_{i}^{(k)}(\tau)\right)^{2}-\left(\widetilde{\theta}_{i}^{(k-1)}(\tau)\right)^{2}\right] d \tau \\
& +\frac{N a \gamma}{2 q_{1}} \int_{0}^{t}\left[\left(\widetilde{\eta}^{(k)}(\tau)\right)^{2}-\left(\widetilde{\eta}^{(k-1)}(\tau)\right)^{2}\right] d \tau .
\end{aligned}
$$

Using Assumption 5 and (14), we have

$$
\begin{aligned}
V^{(k)}(t)= & \int_{0}^{t} \dot{V}^{(k)}(\tau) d \tau+V^{(k)}(0)=\int_{0}^{t} \dot{V}^{(k)}(\tau) d \tau \\
\leq & -c \sum_{i=1}^{N} \int_{0}^{t}\left(\mathbf{e}_{i}^{(k)}(\tau)\right)^{T} \mathbf{e}_{i}^{(k)}(\tau) d \tau \\
& +\sum_{i=1}^{N} \int_{0}^{t} \widetilde{\theta}_{i}^{(k)}(\tau)\left(\mathbf{e}_{i}^{(k)}(\tau)\right)^{T} \boldsymbol{\xi}_{i}\left(\mathbf{x}_{i}^{(k)}(\tau)\right) d \tau \\
& +N a \gamma \sum_{i=1}^{N} \int_{0}^{t} \widetilde{\eta}^{(k)}(\tau)\left(\mathbf{e}_{i}^{(k)}(\tau)\right)^{T} \mathbf{e}_{i}^{(k)}(\tau) d \tau .
\end{aligned}
$$

Using the algebraic relation $(a-b)^{2}-(a-c)^{2}=-2(a-$ $b)(b-c)-(b-c)^{2}$ and the adaptive laws (15), we get

$$
\begin{aligned}
& \frac{1}{2 q_{i 0}} \int_{0}^{t}\left[\left(\widetilde{\theta}_{i}^{(k)}(\tau)\right)^{2}-\left(\widetilde{\theta}_{i}^{(k-1)}(\tau)\right)^{2}\right] d \tau \\
& =\frac{1}{2 q_{i 0}} \int_{0}^{t}\left[-2 \widetilde{\theta}_{i}^{(k)}(\tau)\left(\widehat{\theta}_{i}^{(k)}(\tau)-\widehat{\theta}_{i}^{(k-1)}(\tau)\right)\right. \\
& \left.-\left(\widehat{\theta}_{i}^{(k)}(\tau)-\widehat{\theta}_{i}^{(k-1)}(\tau)\right)^{2}\right] d \tau \\
& =\frac{1}{q_{i 0}} \int_{0}^{t}\left[\theta_{i}(\tau)-\operatorname{sat}\left(\hat{\theta}_{i}^{*(k)}(\tau)\right)\right] \\
& \times\left[\widehat{\theta}_{i}^{*(k)}(\tau)-\operatorname{sat}\left(\widehat{\theta}_{i}^{*(k)}(\tau)\right)\right] d \tau \\
& -\int_{0}^{t} \widetilde{\theta}_{i}^{(k)}(\tau)\left(\boldsymbol{\xi}_{i}\left(\mathbf{x}_{i}^{(k)}(\tau)\right)\right)^{T} \mathbf{e}_{i}^{(k)}(\tau) d \tau \\
& -\frac{1}{2 q_{i 0}} \int_{0}^{t}\left(\widehat{\theta}_{i}^{(k)}(\tau)-\widehat{\theta}_{i}^{(k-1)}(\tau)\right)^{2} d \tau, \\
& \frac{N a \gamma}{2 q_{1}} \int_{0}^{t}\left[\left(\widetilde{\eta}^{(k)}(\tau)\right)^{2}-\left(\widetilde{\eta}^{(k-1)}(\tau)\right)^{2}\right] d \tau \\
& =\frac{N a \gamma}{2 q_{1}} \int_{0}^{t}\left[-2 \widetilde{\eta}^{(k)}(\tau)\left(\hat{\eta}^{(k)}(\tau)-\widehat{\eta}^{(k-1)}(\tau)\right)\right. \\
& \left.-\left(\widehat{\eta}^{(k)}(\tau)-\widehat{\eta}^{(k-1)}(\tau)\right)^{2}\right] d \tau \\
& =\frac{N a \gamma}{q_{1}} \int_{0}^{t}\left[\eta(\tau)-\operatorname{sat}\left(\hat{\eta}^{*(k)}(\tau)\right)\right] \\
& \times\left[\widehat{\eta}^{*(k)}(\tau)-\operatorname{sat}\left(\widehat{\eta}^{*(k)}(\tau)\right)\right] d \tau \\
& -\operatorname{Na\gamma } \sum_{i=1}^{N} \int_{0}^{t} \tilde{\eta}^{(k)}(\tau)\left(\mathbf{e}_{i}^{(k)}(\tau)\right)^{T} \mathbf{e}_{i}^{(k)}(\tau) d \tau \\
& -\frac{N a \gamma}{2 q_{1}} \int_{0}^{t}\left(\hat{\eta}^{(k)}(\tau)-\hat{\eta}^{(k-1)}(\tau)\right)^{2} d \tau .
\end{aligned}
$$

According to Lemma 9 and by carefully calculating, we have

$$
\begin{aligned}
& \frac{1}{2 q_{i 0}} \int_{0}^{t}\left[\left(\widetilde{\theta}_{i}^{(k)}(\tau)\right)^{2}-\left(\widetilde{\theta}_{i}^{(k-1)}(\tau)\right)^{2}\right] d \tau \\
& \quad \leq-\int_{0}^{t} \widetilde{\theta}_{i}^{(k)}(\tau)\left(\boldsymbol{\xi}_{i}\left(\mathbf{x}_{i}^{(k)}(\tau)\right)\right)^{T} \mathbf{e}_{i}^{(k)}(\tau) d \tau, \\
& \frac{N a \gamma}{2 q_{1}} \int_{0}^{t}\left[\left(\widetilde{\eta}^{(k)}(\tau)\right)^{2}-\left(\widetilde{\eta}^{(k-1)}(\tau)\right)^{2}\right] d \tau \\
& \quad \leq-N a \gamma \sum_{i=1}^{N} \int_{0}^{t} \widetilde{\eta}^{(k)}(\tau)\left(\mathbf{e}_{i}^{(k)}(\tau)\right)^{T} \mathbf{e}_{i}^{(k)}(\tau) d \tau .
\end{aligned}
$$


Substituting (19) and (21) into (18) yields

$$
\begin{aligned}
\Delta W^{(k)}(t) & \leq-c \sum_{i=1}^{N} \int_{0}^{t}\left(\mathbf{e}_{i}^{(k)}(\tau)\right)^{T} \mathbf{e}_{i}^{(k)}(\tau) d \tau-V^{(k-1)}(t) \\
& \leq-c \sum_{i=1}^{N} \int_{0}^{t}\left(\mathbf{e}_{i}^{(k)}(\tau)\right)^{T} \mathbf{e}_{i}^{(k)}(\tau) d \tau
\end{aligned}
$$

When $t=T$, inequality (22) is expressed as

$$
\Delta W^{(k)}(T) \leq-c \sum_{i=1}^{N} \int_{0}^{T}\left(\mathbf{e}_{i}^{(k)}(\tau)\right)^{T} \mathbf{e}_{i}^{(k)}(\tau) d \tau .
$$

Secondly, we prove that all signals in the closed-loop system are bounded. Using (17) and choosing $k=0$, we have

$$
\begin{aligned}
W^{(0)}(t)= & V^{(0)}(t)+\sum_{i=1}^{N} \frac{1}{2 q_{i 0}} \int_{0}^{t}\left(\widetilde{\theta}_{i}^{(0)}(\tau)\right)^{2} d \tau \\
& +\frac{N a \gamma}{2 q_{1}} \int_{0}^{t}\left(\widetilde{\eta}^{(0)}(\tau)\right)^{2} d \tau .
\end{aligned}
$$

The derivative of $W^{(0)}(t)$ satisfies

$$
\begin{aligned}
\dot{W}^{(0)}(t)= & \dot{V}^{(0)}(t)+\sum_{i=1}^{N} \frac{1}{2 q_{i 0}}\left(\widetilde{\theta}_{i}^{(0)}(t)\right)^{2}+\frac{N a \gamma}{2 q_{1}}\left(\widetilde{\eta}^{(0)}(t)\right)^{2} \\
\leq & \sum_{i=1}^{N} \frac{1}{2 q_{i 0}}\left(\widetilde{\theta}_{i}^{(0)}(t)\right)^{2}+\frac{N a \gamma}{2 q_{1}}\left(\widetilde{\eta}^{(0)}(t)\right)^{2} \\
& +\sum_{i=1}^{N} \widetilde{\theta}_{i}^{(0)}(t)\left(\mathbf{e}_{i}^{(0)}(t)\right)^{T} \xi_{i}\left(\mathbf{x}_{i}^{(0)}(t)\right) \\
& +N a \gamma \sum_{i=1}^{N} \widetilde{\eta}^{(0)}(t)\left(\mathbf{e}_{i}^{(0)}(t)\right)^{T} \mathbf{e}_{i}^{(0)}(t) \\
& -c \sum_{i=1}^{N}\left(\mathbf{e}_{i}^{(0)}(t)\right)^{T} \mathbf{e}_{i}^{(0)}(t)
\end{aligned}
$$

According to the adaptive learning laws (15), one obtains

$$
\begin{aligned}
\sum_{i=1}^{N} \frac{1}{2 q_{i 0}}\left(\widetilde{\theta}_{i}^{(0)}(t)\right)^{2}+\frac{N a \gamma}{2 q_{1}}\left(\widetilde{\eta}^{(0)}(t)\right)^{2} \\
\leq-\sum_{i=1}^{N} \widetilde{\theta}_{i}^{(0)}(t)\left(\boldsymbol{\xi}_{i}\left(\mathbf{x}_{i}^{(0)}(t)\right)\right)^{T} \mathbf{e}_{i}^{(0)}(t) \\
\quad-N a \gamma \sum_{i=1}^{N} \widetilde{\eta}^{(0)}(t)\left(\mathbf{e}_{i}^{(0)}(t)\right)^{T} \mathbf{e}_{i}^{(0)}(t) \\
+\sum_{i=1}^{N} \frac{1}{2 q_{i 0}}\left(\theta_{i}(t)\right)^{2}+\frac{N a \gamma}{2 q_{1}}(\eta(t))^{2} .
\end{aligned}
$$

Substituting (26) into (25) yields

$$
\dot{W}^{(0)}(t) \leq \sum_{i=1}^{N} \frac{1}{2 q_{i 0}}\left(\theta_{i}(t)\right)^{2}+\frac{N a \gamma}{2 q_{1}}(\eta(t))^{2} .
$$

Because of the continuity of $\theta_{i}(t)$ and $\eta(t)$ on $[0, T]$, a positive constant $M$ exists so that

$$
\begin{array}{r}
\dot{W}^{(0)}(t) \leq \sum_{i=1}^{N} \frac{1}{2 q_{i 0}}\left(\theta_{i}(t)\right)^{2}+\frac{N a \gamma}{2 q_{1}}(\eta(t))^{2} \leq M, \\
t \in[0, T] .
\end{array}
$$

Using Assumption 5, we have $W^{(0)}(0)=0$. Applying $W^{(0)}(0)=0$ into inequality $(28)$ yields

$$
W^{(0)}(t) \leq \int_{0}^{t} M d \tau \leq M T<\infty, \quad t \in[0, T],
$$

which shows that $W^{(0)}(t)$ is bounded on $[0, T]$.

Moreover, by invoking the definition of $W^{(k)}(t)$ and applying inequality (22), we have

$$
\begin{aligned}
W^{(k)}(t)= & \Delta W^{(k)}(t)+W^{(k-1)}(t) \leq W^{(k-1)}(t) \\
& -c \sum_{i=1}^{N} \int_{0}^{t}\left(\mathbf{e}_{i}^{(k)}(\tau)\right)^{T} \mathbf{e}_{i}^{(k)}(\tau) d \tau \leq W^{(k-1)}(t) .
\end{aligned}
$$

Therefore,

$$
W^{(k)}(t) \leq W^{(k-1)}(t) \leq W^{(k-1)}(T), \quad t \in[0, T] .
$$

It also follows from (30) that

$$
W^{(k)}(T) \leq W^{(k-1)}(T)-c \sum_{i=1}^{N} \int_{0}^{T}\left(\mathbf{e}_{i}^{(k)}(\tau)\right)^{T} \mathbf{e}_{i}^{(k)}(\tau) d \tau,
$$

which gives rise to

$$
W^{(k)}(T) \leq W^{(k-1)}(T), \quad k=1,2,3, \ldots ;
$$

$W^{(k)}(T)$ is thus a nonincreasing sequence. It then follows from (29), (31), and (33) that $W^{(k)}(t)(t \in[0, T])$ is uniformly bounded with respect to $k$. In addition, by invoking the definition of $W^{(k)}(t), \mathbf{e}_{i}^{(k)}(t)$ is uniformly bounded with respect to $k$. Therefore, $\mathbf{x}_{i}^{(k)}(t)$ and $\boldsymbol{\xi}_{i}\left(\mathbf{x}_{i}^{(k)}(t)\right)$ are obviously uniformly bounded with respect to $k$. According to (15), we can prove that $\widehat{\theta}_{i}^{(k)}(t)$ and $\widehat{\eta}^{(k)}(t)$ are uniformly bounded with respect to $k$. Hence, all signals in the closed-loop system are bounded.

Finally, we prove the convergence of tracking error sequence. It follows from (32) that

$$
\sum_{i=1}^{N} \int_{0}^{T}\left(\mathbf{e}_{i}^{(k)}(\tau)\right)^{T} \mathbf{e}_{i}^{(k)}(\tau) d \tau \leq \frac{1}{c}\left[W^{(k-1)}(T)-W^{(k)}(T)\right] .
$$

Since $W^{(k)}(T)$ is monotone decreasing but lower bounded, $W^{(k)}(T)$ converges. Therefore,

$$
\lim _{k \rightarrow \infty} \sum_{i=1}^{N} \int_{0}^{T}\left(\mathbf{e}_{i}^{(k)}(\tau)\right)^{T} \mathbf{e}_{i}^{(k)}(\tau) d \tau=0 ;
$$




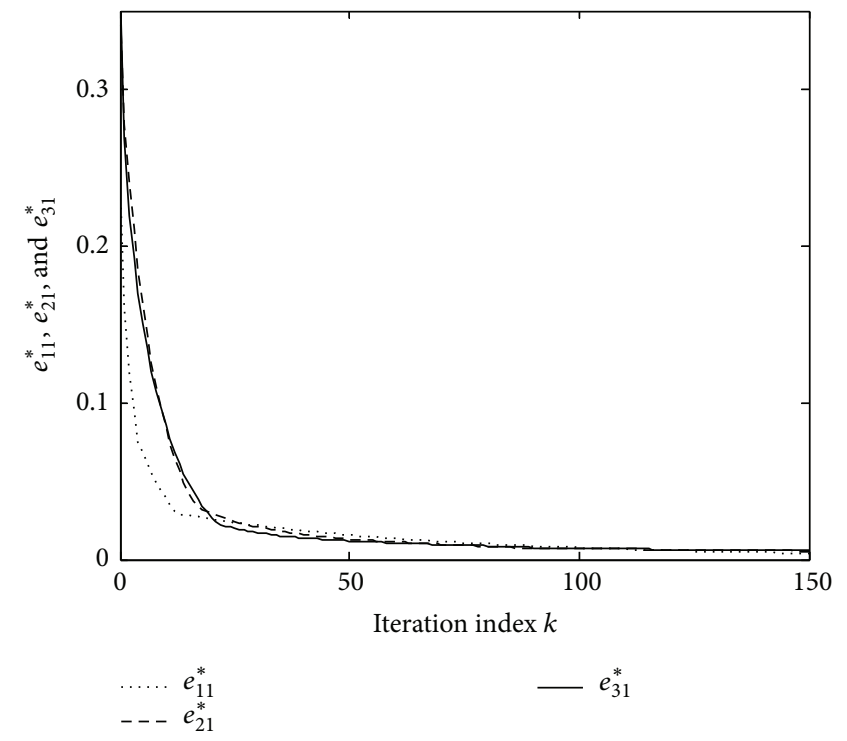

(a)

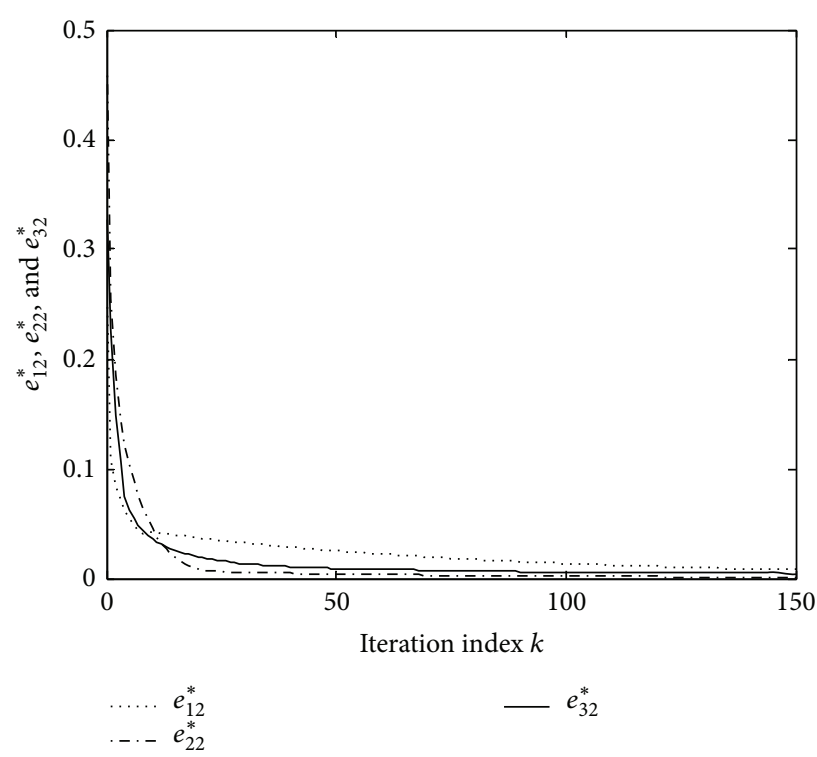

(b)

FIGURE 1: The change of $e_{i j}^{*}=\max _{t \in[0,50]}\left|e_{i j}^{(k)}(t)\right|(i=1,2,3 ; j=1,2)$ with iteration index $k$.

then,

$$
\lim _{k \rightarrow \infty} \int_{0}^{T}\left(\mathbf{e}_{i}^{(k)}(\tau)\right)^{T} \mathbf{e}_{i}^{(k)}(\tau) d \tau=0
$$

According to Lemma 7, we get $\lim _{k \rightarrow \infty} \mathbf{e}_{i}^{(k)}(t)=0$, which shows that the tracking error sequence of each node converges.

Remark 12. From the result of the theorem, we get that the state of each node tracks the desired trajectory over a given fixed time interval as the iteration index tends to infinity. Hence, it is also said that the synchronization objective for complex dynamical networks is obtained in the iteration domain.

\section{Simulation Example}

In this section, a numerical example is given to show the effectiveness of the designed method.

Example 1. Consider a complex dynamical network with repetitive operation over a fixed time interval $[0,50]$ as follows:

$$
\begin{array}{r}
\dot{\mathbf{x}}_{i}(t)=\theta_{i}(t) \boldsymbol{\xi}_{i}\left(\mathbf{x}_{i}(t)\right)+\eta(t) \sum_{j=1}^{3} a_{i j} \boldsymbol{\Gamma} \mathbf{x}_{j}(t)+\mathbf{u}_{i}(t), \\
i=1,2,3 .
\end{array}
$$

The parameters in the network (37) are

$$
\begin{gathered}
A=\left(a_{i j}\right)_{3 \times 3}=\left(\begin{array}{ccc}
-1 & 1 & 0 \\
1 & -2 & 1 \\
0 & 1 & -1
\end{array}\right), \quad \Gamma=\left(\begin{array}{ll}
1 & 0 \\
0 & 1
\end{array}\right), \\
\xi_{1}\left(\mathbf{x}_{1}\right)=\left(\begin{array}{c}
-2.5 x_{11}^{2}+1.3 x_{12} \\
x_{11} x_{12}
\end{array}\right), \\
\xi_{2}\left(\mathbf{x}_{2}\right)=\left(\begin{array}{c}
-x_{21}+1.3 x_{22}^{2} \\
2 x_{21}-x_{21}^{2} x_{22}
\end{array}\right), \\
\xi_{3}\left(\mathbf{x}_{3}\right)=\left(\begin{array}{c}
-x_{31}+1.5 x_{32} \\
-1.2 x_{32}^{2}+x_{31} x_{32}
\end{array}\right), \quad \theta_{1}(t)=-0.5 \sin t, \\
\theta_{2}(t)=1-0.6 \sin t, \quad \theta_{3}(t)=-1.1 \cos t, \\
\eta(t)=1+\cos t .
\end{gathered}
$$

The desired trajectory is $\mathbf{s}(t)=(\sin (2 \pi t / 50), 1+\sin$ $(2 \pi t / 50))^{T}$. Based on the dynamical equation (37), we have $N=3, a=2, \gamma=1$, and $N a \gamma=6$. The design parameters are selected as follows:

$$
\begin{gathered}
q_{10}=0.6, \quad q_{20}=0.7, \quad q_{30}=0.5, \\
q_{1}=0.8, \quad c=1 .
\end{gathered}
$$

In the simulation, we select $\{-2,2\}$ as the lower and upper bounds of the saturation function, so that the time-varying parameters are within the bounds. Because $\mathbf{s}(0)=(0,1)^{T}$, we select $\mathbf{x}_{i}^{(k)}(0)=(0,1)^{T}(i=1,2,3)$ as the initial state. 


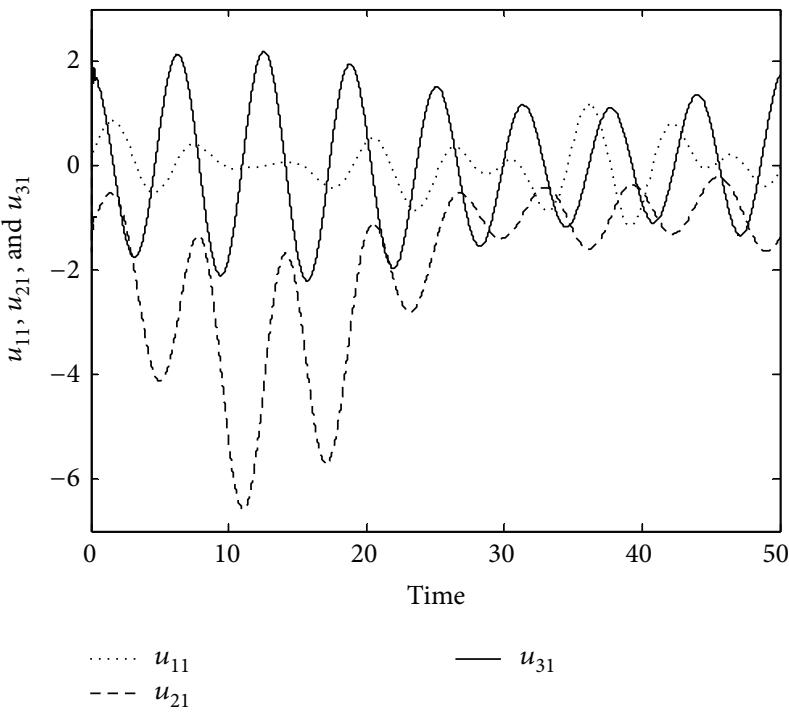

(a)

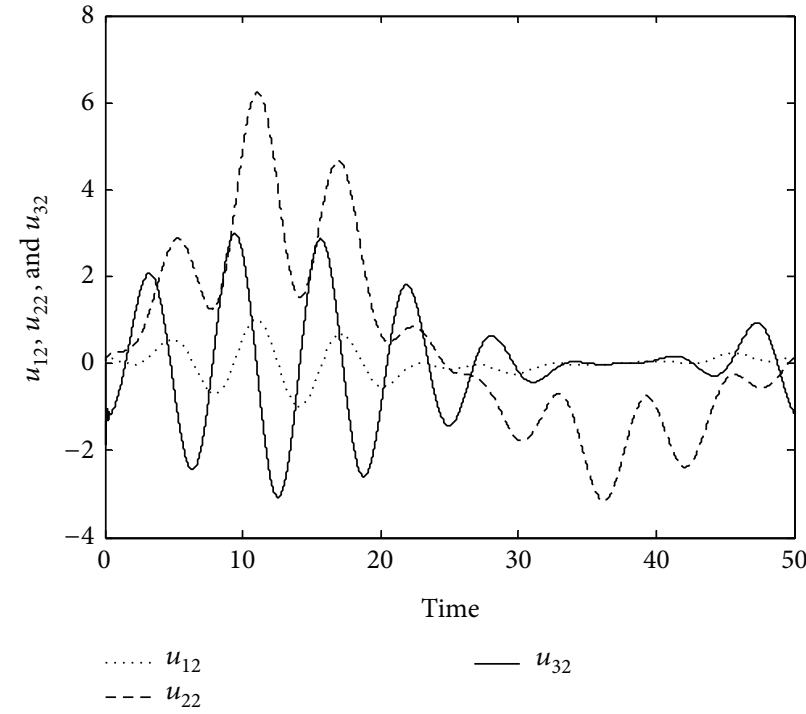

(b)

FIGURE 2: The curve of control inputs $u_{i j}=u_{i j}^{(k)}(t)(i=1,2,3 ; j=1,2)$ as iteration index $k=150$.

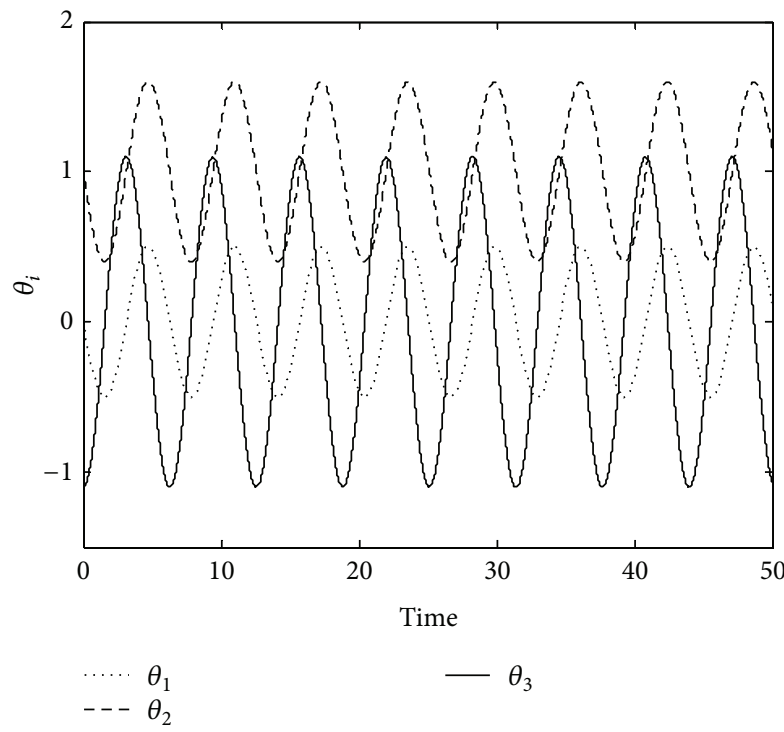

(a)

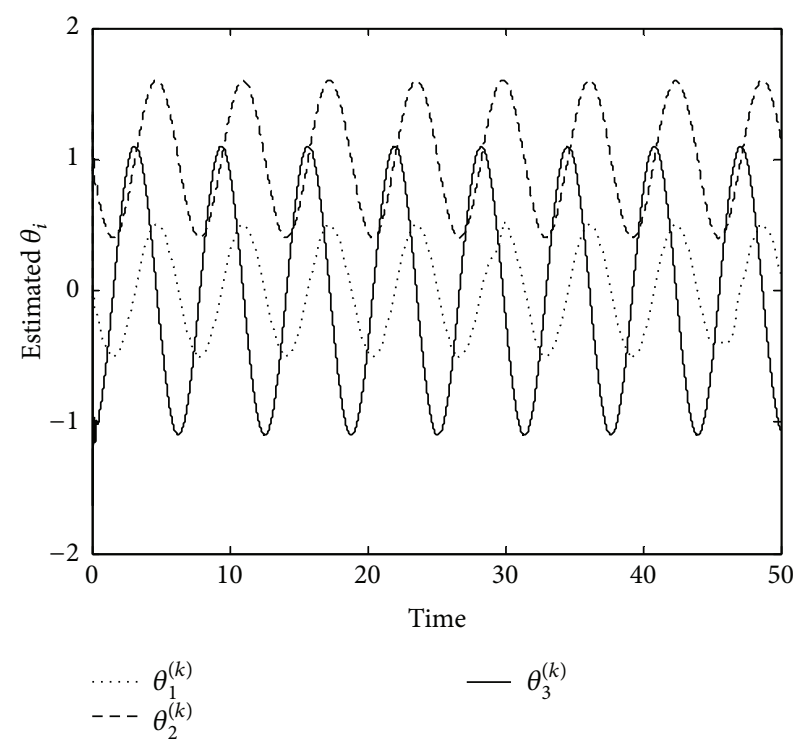

(b)

FIGURE 3: The evaluations of parameters $\theta_{i}(t)$ and $\theta_{i}^{(k)}=\hat{\theta}_{i}^{(k)}(t)(i=1,2,3)$ as iteration index $k=150$.

The simulation results are shown in the Figures 14. Figure 1 shows that a perfect tracking performance is achieved as the iteration index increases. Figure 2 depicts the curves of control inputs. Figure 3 shows the evolutions of $\theta_{i}(t)$ and $\widehat{\theta}_{i}^{(k)}(t)$ as iteration index $k=150$. Figure 4 shows the evolutions of $\eta(t)$ and $\hat{\eta}^{(k)}(t)$ as iteration index $k=$ 150. Obviously, the controllers and estimated parameters are bounded on $[0,50]$.

Remark 13. From the simulation results shown in the above figures, we find the estimated parameters of $\theta_{i}(t)$ identical to the true values, which shows that the unknown terms in the dynamical equation of each node can be identified very well. However, the estimated parameter of $\eta(t)$ is different from the true value. This is because of the simple feedback controller (9) added in each node. In this paper, we analyze the network topology of complex dynamical networks and design the controller without the precise information of the network topology, and then we get the perfect tracking performance. Thus, the control objective can be achieved even if the time-varying coupling strength was not identified very well. 


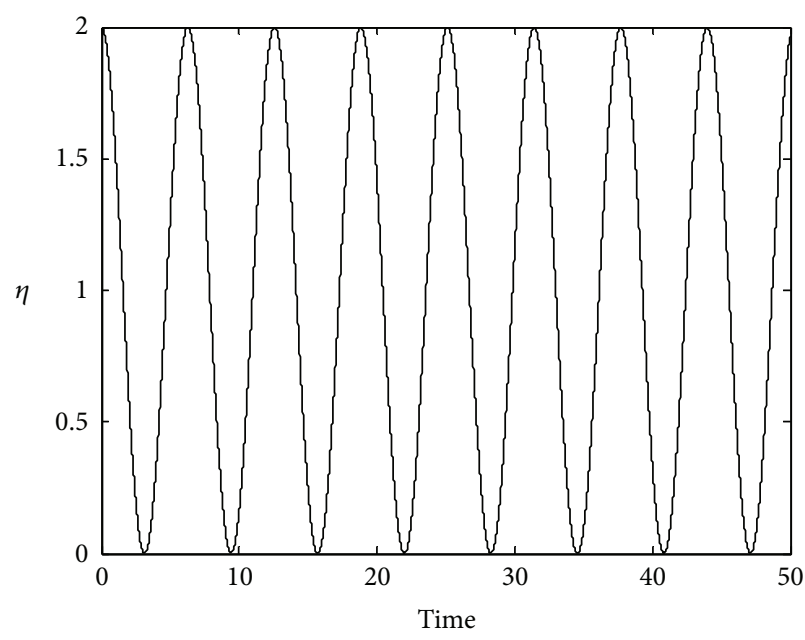

(a)

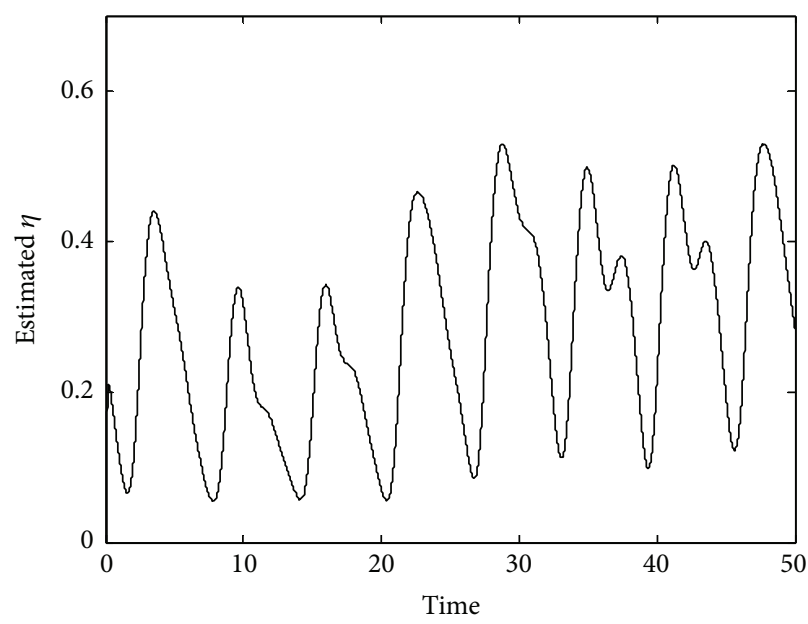

(b)

FIGURE 4: The evaluations of parameters $\eta(t)$ and $\hat{\eta}^{(k)}(t)$ as iteration index $k=150$.

\section{Conclusions}

In this paper, for a complex dynamical network with repetitive operation over a fixed time interval, we propose a new adaptive iterative learning control scheme to ensure that the state of each node tracks the desired trajectory as the iteration index $k$ tends to infinity. In this context, it is also said that the synchronization for complex dynamical networks is achieved in the iteration domain. The simulation example verifies the theoretical results.

\section{Conflict of Interests}

The authors declare that there is no conflict of interests regarding the publication of this paper.

\section{Acknowledgments}

This work was supported by the National Natural Science Foundation of China under Grant no. 60974139 and the Foundation of Ministry of Education of China under Grants no. JY0300137002 and no. 20130203110021.

\section{References}

[1] S. Arimoto, S. Kawamura, and F. Miyazaki, "Bettering operation of robots by learning," Journal of Robotic Systems, vol. 1, no. 2, pp. 123-140, 1984.

[2] C.-J. Chien, C.-T. Hsu, and C.-Y. Yao, "Fuzzy system-based adaptive iterative learning control for nonlinear plants with initial state errors," IEEE Transactions on Fuzzy Systems, vol. 12, no. 5, pp. 724-732, 2004.

[3] J.-X. Xu and Y. Tan, "A composite energy function-based learning control approach for nonlinear systems with timevarying parametric uncertainties," IEEE Transactions on Automatic Control, vol. 47, no. 11, pp. 1940-1945, 2002.

[4] A. Tayebi and C.-J. Chien, "A unified adaptive iterative learning control framework for uncertain nonlinear systems," IEEE
Transactions on Automatic Control, vol. 52, no. 10, pp. 1907-1913, 2007.

[5] W. Chen and L. Zhang, "Adaptive Iterative learning control for nonlinearly parameterized systems with unknown timevarying delays," International Journal of Control, Automation and Systems, vol. 8, no. 2, pp. 177-186, 2010.

[6] M. Sun, "A Barbalat-like lemma with its application to learning control," IEEE Transactions on Automatic Control, vol. 54, no. 9, pp. 2222-2225, 2009.

[7] J.-X. Xu and J. Xu, "On iterative learning from different tracking tasks in the presence of time-varying uncertainties," IEEE Transactions on Systems, Man, and Cybernetics B: Cybernetics, vol. 34, no. 1, pp. 589-597, 2004.

[8] R. Chi, Z. Hou, and J. Xu, "Adaptive ILC for a class of discretetime systems with iteration-varying trajectory and random initial condition," Automatica, vol. 44, no. 8, pp. 2207-2213, 2008.

[9] W. Yan and M. Sun, "Adaptive iterative learning control of discrete-time varying systems with unknown control direction," International Journal of Adaptive Control and Signal Processing, vol. 27, no. 4, pp. 340-348, 2013.

[10] D. Li and J.-M. Li, "Adaptive iterative learning control for nonlinearly parameterized systems with unknown time-varying delay and unknown control direction," International Journal of Automation and Computing, vol. 9, no. 6, pp. 578-586, 2012.

[11] S. H. Strogatz, "Exploring complex networks," Nature, vol. 410, no. 6825 , pp. 268-276, 2001.

[12] M. E. J. Newman, "The structure and function of complex networks," SIAM Review, vol. 45, no. 2, pp. 167-256, 2003.

[13] D. J. Watts and S. H. Strogatz, "Collective dynamics of 'smallworld' networks," Nature, vol. 393, no. 6684, pp. 440-442, 1998.

[14] X. F. Wang and G. Chen, "Complex networks: small-world, scale-free and beyond," IEEE Circuits and Systems Magazine, vol. 3, no. 1, pp. 6-20, 2003.

[15] Y. Chen, W. Yu, F. Li, and S. Feng, "Synchronization of complex networks with impulsive control and disconnected topology," IEEE Transactions on Circuits and Systems II: Express Briefs, vol. 60, no. 5, pp. 292-296, 2013. 
[16] X. Yang, J. Cao, and J. Lu, "Stochastic synchronization of complex networks with nonidentical nodes via hybrid adaptive and impulsive control," IEEE Transactions on Circuits and Systems I: Regular Papers, vol. 59, no. 2, pp. 371-384, 2012.

[17] J. Zhou, Q. Wu, and L. Xiang, "Pinning complex delayed dynamical networks by a single impulsive controller," IEEE Transactions on Circuits and Systems I: Regular Papers, vol. 58, no. 12, pp. 2882-2893, 2011.

[18] M. Porfiri and M. di Bernardo, "Criteria for global pinningcontrollability of complex networks," Automatica, vol. 44, no. 12, pp. 3100-3106, 2008.

[19] D. H. Ji, S. C. Jeong, J. H. Park, S. M. Lee, and S. C. Won, "Adaptive lag synchronization for uncertain complex dynamical network with delayed coupling," Applied Mathematics and Computation, vol. 218, no. 9, pp. 4872-4880, 2012.

[20] X. Guo and J. Li, "A new synchronization algorithm for delayed complex dynamical networks via adaptive control approach," Communications in Nonlinear Science and Numerical Simulation, vol. 17, no. 11, pp. 4395-4403, 2012.

[21] X. Guo and J. Li, "Stochastic adaptive synchronization for timevarying complex delayed dynamical networks with heterogeneous nodes," Applied Mathematics and Computation, vol. 222, pp. 381-390, 2013.

[22] X.-Z. Jin and G.-H. Yang, "Adaptive pinning synchronization of a class of nonlinearly coupled complex networks," Communications in Nonlinear Science and Numerical Simulation, vol. 18, no. 2, pp. 316-326, 2013.

[23] Y. Wang and J. Cao, "Cluster synchronization in nonlinearly coupled delayed networks of non-identical dynamic systems," Nonlinear Analysis: Real World Applications, vol. 14, no. 1, pp. 842-851, 2013.

[24] H. Wu, "Decentralised adaptive robust control of uncertain large-scale interconnected systems with multiple time-varying delays," International Journal of Systems Science, vol. 43, no. 10, pp. 1842-1854, 2012.

[25] H. Wu, "Decentralized iterative learning control for a class of large scale interconnected dynamical systems," Journal of Mathematical Analysis and Applications, vol. 327, no. 1, pp. 233$245,2007$. 


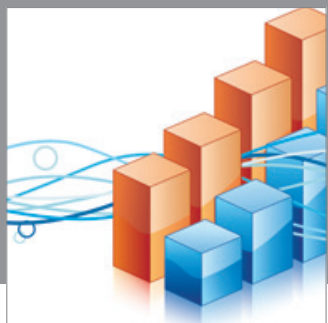

Advances in

Operations Research

mansans

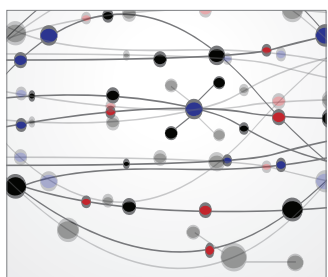

The Scientific World Journal
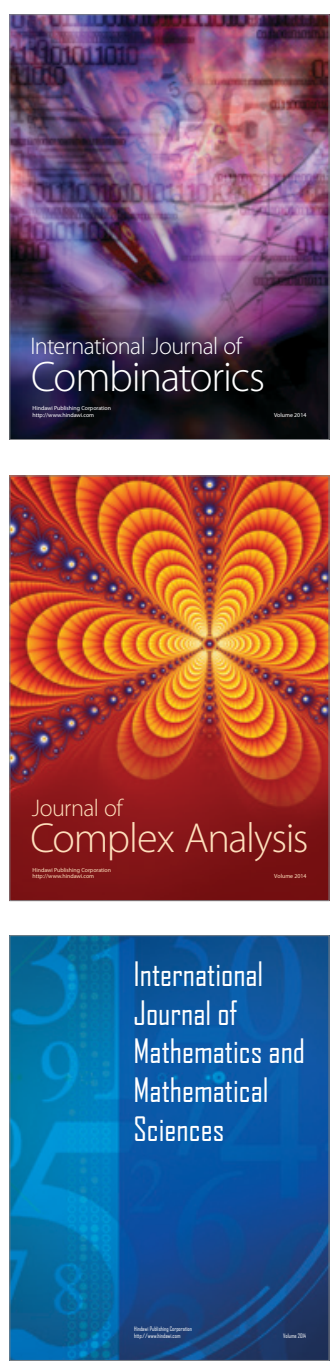
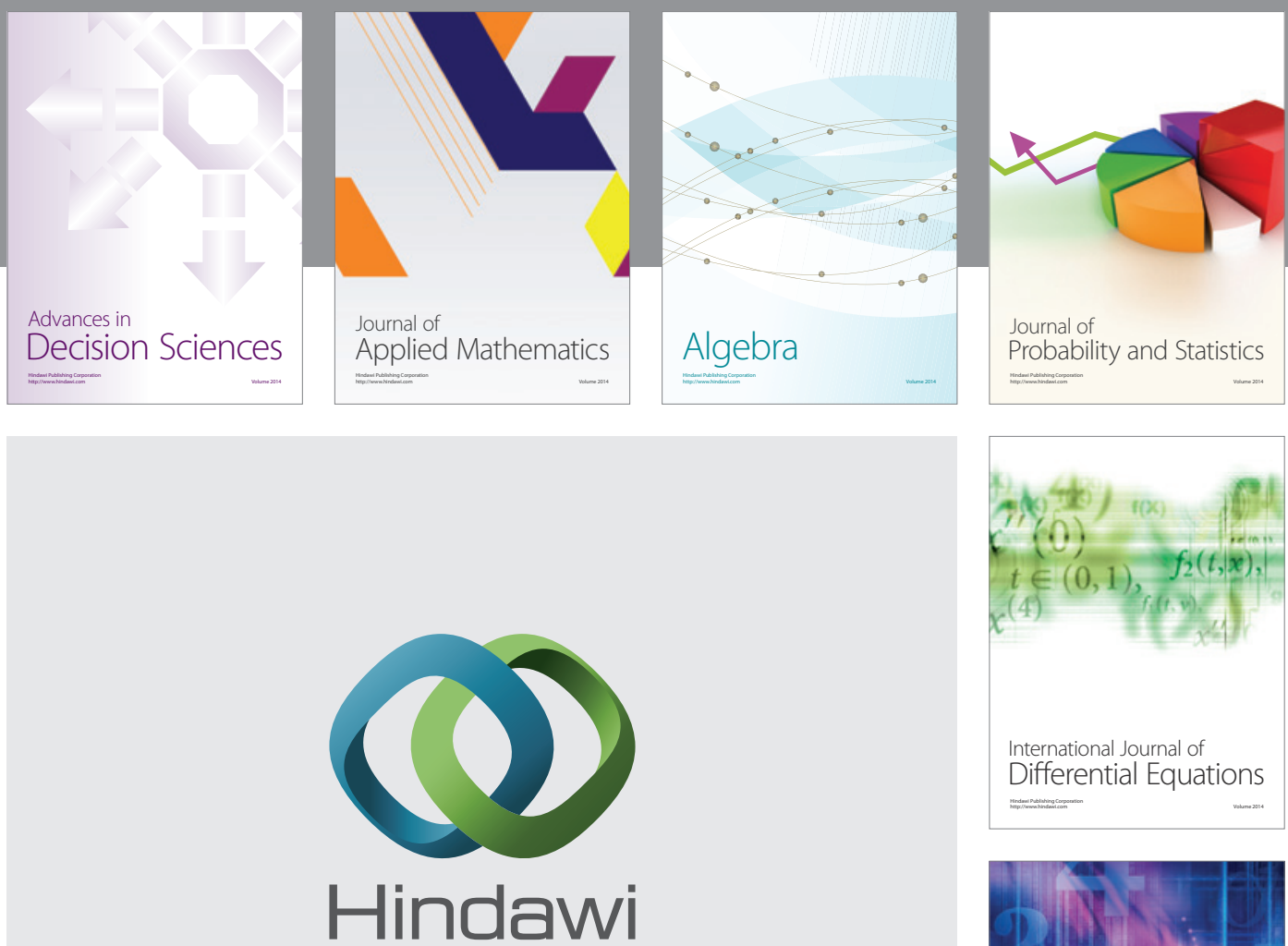

Submit your manuscripts at http://www.hindawi.com
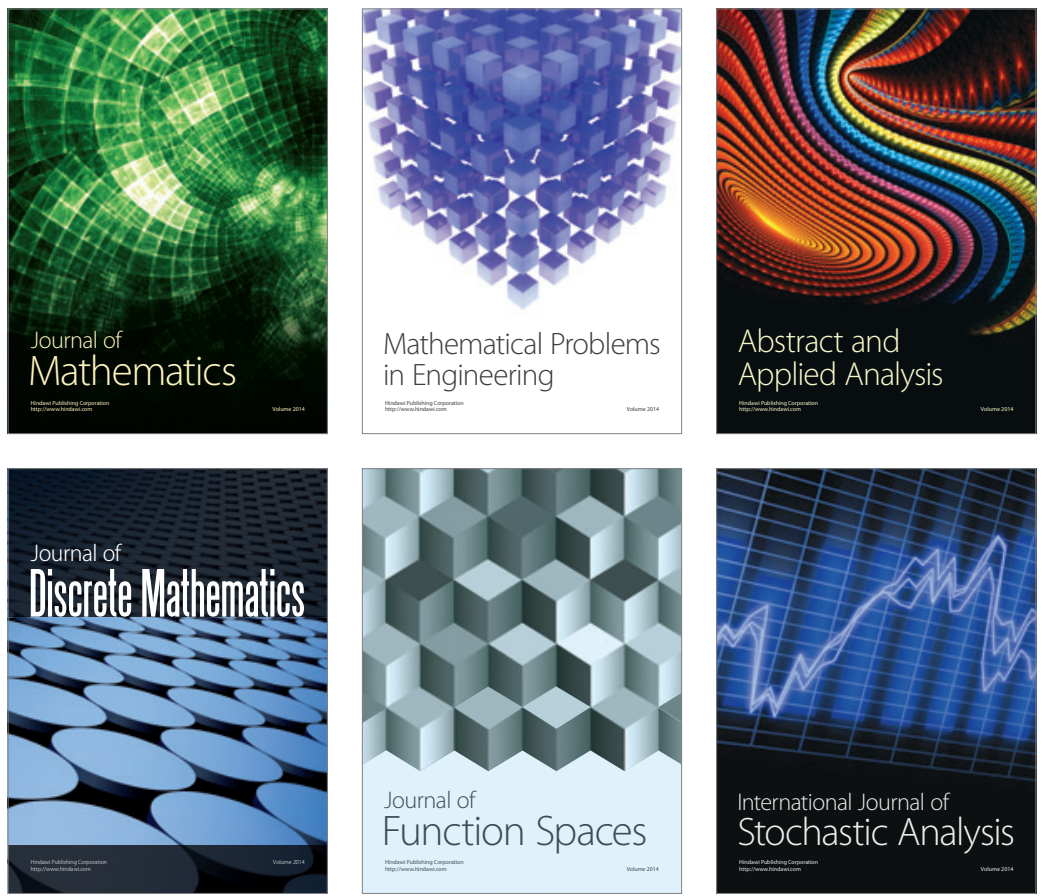

Journal of

Function Spaces

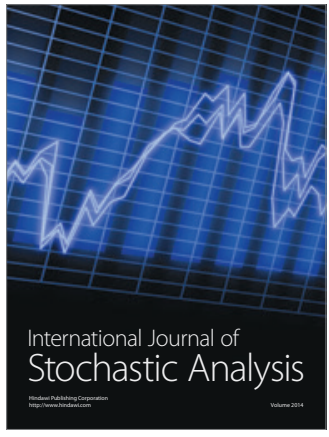

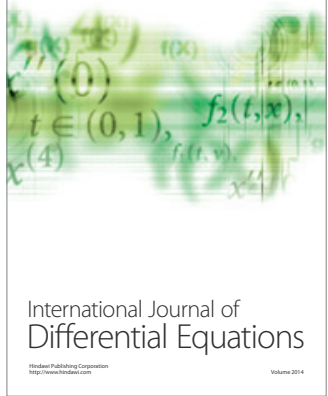
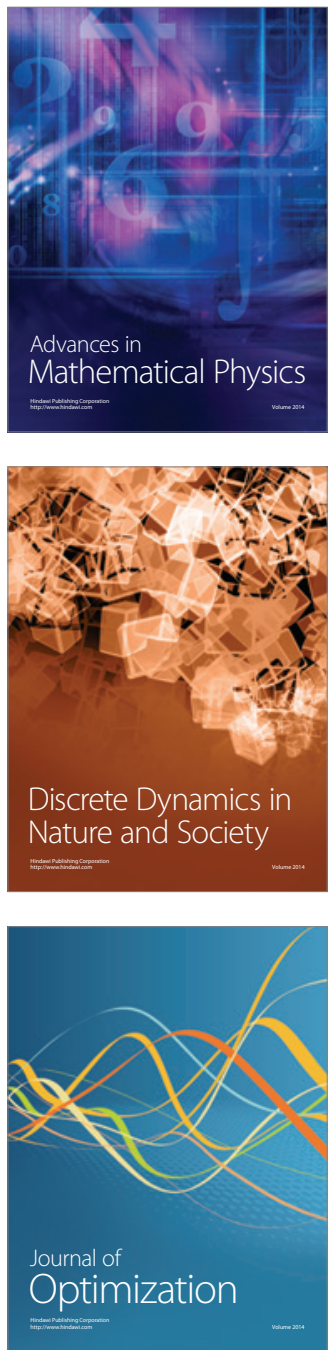